\title{
Thermodynamics and Transport in Microporous Media
}

A renewal request and progress report submitted to the

Department of Energy

Office of Basic Energy Sciences

DOE/ER/13825--8

DE93 004370

September 1992

Principal Investigator:

Science

Contract Number:

Starting Date of third year:

Total Duration:

Corporate Name of University:

Contracting Office:

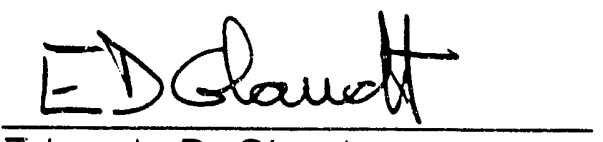

Eduardo D. Glandt

Principal Investigator and Chairman, Department of Chemical Engineering
Professor Eduardo D. Glandt

Department of Chemical Engireering

School of Engineering and Applied

University of Pennsylvania

Philadelphia, PA 19104-6393

DE-FG02-88ER13825

1 January 1993

Three Years

The Trustees of the University of Pennsylvania

Office of Research Administration

Suite 300 Mellon Building/3246

Philadelphia, PA 19104 


\title{
THERMODYNAMICS AND TRANSPORT IN MICROPOROUS MEDIA
}

\author{
A Progress Report the \\ U.S. Department of Energy \\ Office of Basic Energy Sciences
}

by

\author{
Eduardo D. Glandt \\ Department of Chernical Engineering \\ University of Pennsylvania \\ Philadelphia, PA 19104-6393
}

October 1992

DISCLAIMER

\begin{abstract}
This report was prepared as an account of work sponsored by an agency of the United States Government. Neither the United States Gnvernment nor any agency thereof, nor any of their employees, makes any warranty, express or implied, or assumes any legal liability or responsibility for the accuracy, completeness, or usefulness of any information, apparatus, product, or process disclosed, or represents that its use would not infringe privately owned rights. Reference herein to any specific commercial product, process, or service by trade name, trademark, manufacturer, or otherwise does not necessarily constitute or imply its endorsement, recommendation, or favo-ing by the United States Government or any agency thereof. The views and opinions of authors expressed herein do not necessarily state or reflect those of the United States Government or any agency thereof.
\end{abstract}


This report covers the activities sponsored at the University of Pennsylvania under contract No. DE-FG02-88ER13825. The following are some of the principal results for the period October 30, 1991 to July 31, 1992.

The main focus of our work is the modeling of disordered solids (random microporous materials) with attention to two sets of problems:

(1) the effective, transport properties of the solid material, and

(2) the thermodynamic behavior of fluids contained in or adsorbed onto the microporous matrix.

Although we continue our work along both lines, the focus of the present report will be on the second of them, i.e. the thermodynamic properties. This is an area in which we have made truly pioneering contributions.

Fluids occur in micropores almost as frequently as they occur in the bulk. The technological significance of the problem is huge. It encompasses sub-problems as big as adsorption on microporous solids (the most important class), membrane partitioning, chromatographic separations, and capillarity and drying processes, to name just a few. From a thermodynamic stand-point, all of these physical situations fall under the same heading: "fluids and fluid mixtures in an external field," the field being the attraction or simply the confinement caused by the solid.

The state-of-the-art, rigorous approach to describing a fluid in an external field is density functional theory. It is well developed and has been very successful in dealing with a fluid next to a wall, next to a fiber, within a flat slit or within a cylindrical pore, i.e. "well-behaved" inhomogeneities. Density functional theory cannot be applied to the challenging case of disordered houndaries: a packed bed, a sintered solid, or a randomly porous solid of any kind. However, it is imperative to deal with the detailed disordered geometry of the matrix if the characteristic diameter of the pores is comparable to the largest molecular dimension (range of the intermolecular forces, correlation length (e.g. for capillary condensation), radius of gyration (for polymers). This includes many cases when the accessibility of the pores is an issue and it is necessary to distinguish between connected and disconnected areas. 
In Madden and Glandt ( $\mathrm{E}$ : $c$ tribution Functions for Fluids in Random Media," J. Stat. Phys. 51. 537. 1988) we first proposed a totally new approach for the calculation of the properties of fluids in disordered matrices. This theory takes advantage of the similarities -- both real and formal -- between the problem at hand and that of a binary equilibrium mixture in the bulk. The tluid and the random matrix in which it is embedded may indeed be considered as a binary system, only that one of the "components," the obstacles comprising the solid, is quenched, i.e. is not constantly rearranging its configuration in response to the energetic interactions.

In a first series of papers (see, for example Fanti et al., "Fluids in Equilibrium within Disordered Porous Materials. Integral Equation Theory," J. Chem. Phys., 93, 5945, 199()), we applied the Madden-Glandt formalism to highly idealized model systems: tluids of hard spheres embedded within packed beds, sintered beds and fibrous matrices. The theory compares very favorably with computer simulations and established the usefulness of the approach.

Our efforts of the last year have been concentrated on implementing the same treatment for realistic systems. This realism is brought about along two different lines: (a) gas adsorption in model carbons, and (b) polymer partitioning. Our models for disordered activated carbons are the first ones ever proposed that account for the detailed structure of carbon. All previous studies of gas adsorption were invariably based on ad hoc simplifications such as planar, cylindrical or even triangular pores of intinite length "drilled" through blocks of solid carbon. In contrast, we consider the solid as an arrangement of graphitic microcrystals, of size comparable to those determined by $X$-ray scattering and of density equivalent to the pellet density of industrial carbons. We have heen able to reproduce adsorption isotherms for methane over activated carbons almost quantitatively and without using any adjustable parameters. We are now extending the same studies to the case of polar adsorbates, most notably water vapor.

Our study of the partitioning of chain molecules between a bulk phase and the micropores or crevices of a solid matrix (i.e. the first theory of gel-permeation chromatography) is one of the most challenging that we have undertaken to date. Our first studies include not only the case of long chains but also the very related problem of the adsorption of flexible molecular fluids (paraffins such as $\mathrm{C}_{1(\text { ) }}$ ). The RISM formalism of Chandler and coworkers is the state-of-the-art theory for such molecular species in the bulk. We have 
combined the RISM theory with the Madden-Glandt treatment for simple fluids in a random medium. Our preliminary calculations reflect the expected sensitivity of the structure of the chain molecule on the porosity of the solid. The first manuscripts containing such calculations should be ready for submission before the end of the year.

We have also continued our work on the characterization of the microgeometry of solids, their percolation and connectedness properties. In addition to the structures described in previous reports, we have linished a very complete study of the simplest and yet most useful model for microporous materials: the random-pore or "Swiss cheese" model. We have published rigorous analytical results on a number of important properties such as the length of lines at the throats between pairs of pores and the number concentration of cusps at the intersection of three pores. These properties have proven to he of relevance in the estimation of the adsorption capacity of a solid and other related applications.

We have also continued last year's work on irreversible adsorption. Normally, the study of such processes (which approximate phenomena such as protein adsorption) ignores all physical interactions: the adsomption mechanism is defined by an algorithm of successive placement of molecules over a substrate, and no allowance is made (or can be made) for the physics of the system, i.e. the attraction between adsorbent and adsorbate or between adsorbate molecules. We have sought to bring the physics back into the picture by studying a simple yet realistic model for correlated adsorption, which describes the physics of a ballistic-type deposition of the adsorbate. 


\title{
PUBLICATIONS
}

\section{IN PRINT OR IN PRESS}

\author{
A.P. Thompson and E.D. Glandt, "Random Sequential Adsorption in Microporous \\ Materials," J. Colloid Interf. Sci. 146, 63 (1991).
}

A.Ott Weist and E.D. Glandt, "Clustering and Percolation for Dimerizing Penetrable Spheres," J. Chem. Phys. 95. 8365 (1991).

K.R. Matranga, A.L. Myers and E.D. Glandt, "Storage of Natural Gas by Adsorption on Activated Carbon," Chem. Eng. Sci., 47, 1569 (1992).

A.P. Thompson and E.D. Gland, "The Length of Intersection Lines and the Number of Cusps in Assemblies of Interpenetrating Spheres," J. Chem. Phys. 97, 1932 (1992).

K.R. Matranga, A.L Myers and E.D. Glandt, "Molecular Simulation of Adsorbed Natural Gas," Separation Sci. Technol., in press (November 1992).

A.O. Weist and E.D. Glandt, Clustering and Percolation for Dimerizing Adhesive Spheres," J. Chem. Phys., in press ( September 1992).

A.P. Thompson and E.D. Glandt, "Low Coverage Kinetics of Correlated Sequential Adsorption," Phys. Rev. A, in press (October 1992).

\section{SUBMITTED}

A.Ott Wiest and E.D. Glandt, "Equilibrium Polymerization and Gelation. I. IntegralEquation Theory," submitted to J. Chem. Phys. (1992).

A.Ou Wiest and E.D Glandt, "Equilibrium Polymerization and Gelation.

II. Percolation of Penetrable Chains," submitted to J. Chem. Phys. (1992). 


\section{IN PREPARATION}

E.A. Segarra and E.D. Glandt, "Model Activated Carbons: I. Microstructure and Methane Adsorption," ready to be submitted to Langmuir.

A.O. Wiest and E.D. Glandt, "Equilibrium Polymerization and Gelation. III. Hydrogen Bonded Chains," to be submitted to .J. Chem. Phys. (1992).

A.Ott Wiest and E.D. Glandt. "Equilibrium Polymerization and Gelation. IV. CrossLinked Chains," to be submitted to J. Chem. Phys. (1992).

E.A. Segarra and E.D. Glandt, "Model Lamellar Materials: Microstructure and Sulute Partitioning," to he submitted to J. Colloid Interf. Sci. (1992). 

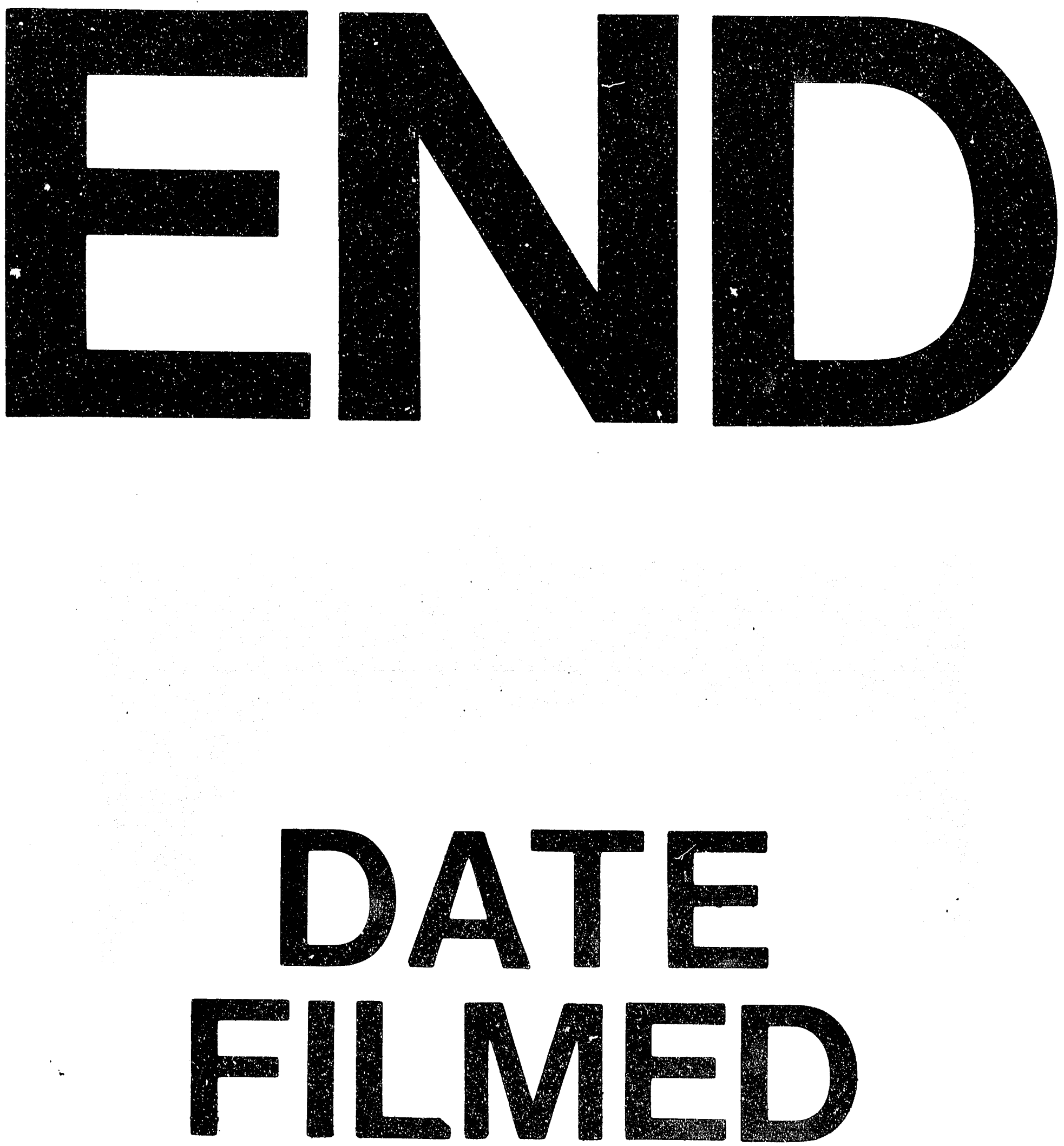

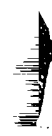
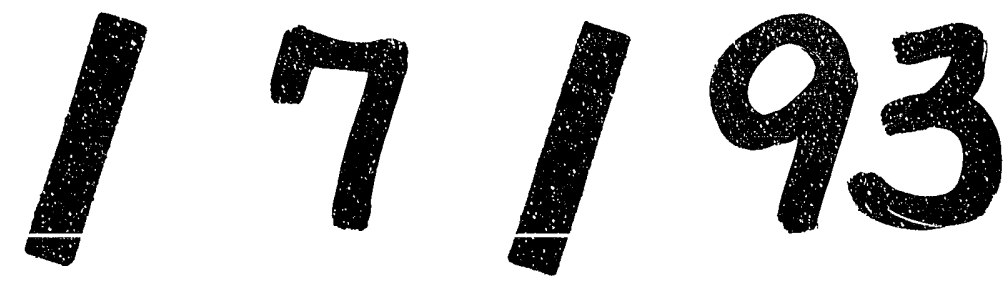


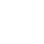

